\title{
Features and outcome in meningococcal disease presenting with maculopapular rash
}

Institute of Child Health, Royal Liverpool Children's Hospital, Alder Hey, Eaton Road, Liverpool L12 2AP O Marzouk A P J Thomson

J A Sills

F Harris

Department of Medical Microbiology, University of Liverpool C A Hart

Correspondence to: Dr Marzouk.

Accepted 15 November 1990

\author{
O Marzouk, A P J Thomson, J A Sills, C A Hart, F Harris
}

\begin{abstract}
Sixty nine patients with meningococcal disease some of whom presented with a maculopapular rash were entered in a prospective multicentre study. The clinical and laboratory features of children presenting with maculopapular rashes were compared with those of children presenting with typical haemorrhagic rashes. Of the 69 children $26(38 \%)$ developed maculopapular rashes; nine $(13 \%)$ had a maculopapular rash only, and the remaining 17 had a mixed maculopapular-purpuric rash. Twelve of the $17(7 \%)$ had less than 12 petechiae. Children with maculopapular rashes had significantly higher platelet counts (median 294 compared with $243 \times 10^{9} / 1$ ), and plasma total haemolytic complement activity (80.5 compared with $65.0 \mathrm{U} / \mathrm{ml}$ ) and significantly lower Glasgow meningococcal septicaemia prognostic scores ( 2.5 compared with 5.5) than those with purpuric rashes on admission. There were no significant differences between the groups in mortality, white cell count or absolute neutrophil count on admission, or $\mathbf{C}$ reactive protein concentration.

Meningococcal disease can present with a maculopapular rash alone but this does not necessarily mean that the disease is less severe.
\end{abstract}

The haemorrhagic rash seen in meningococcal disease is an important diagnostic and prognostic feature that varies in distribution and size from petechiae to ecchymoses. ${ }^{1}$ There have been isolated case reports of meningococcal disease presenting with a maculopapular rash, ${ }^{23}$ but the incidence has not been established in a prospective series.

In this prospective study we have compared the clinical and laboratory features and outcome of children who presented with maculopapular rashes with those of children who presented with classic rashes in meningococcal disease.

\section{Patients and methods}

During the year ending 14 November 1989, 69 consecutive children who presented at the Royal Liverpool Children's Hospitals (Alder Hey and Myrtle Street) and the paediatric units at Whiston, St Helens, Arrowe Park, and the Countess of Chester Hospitals with meningococcal disease were included in a prospective study.

On admission all children were seen by one of us $(O M)$. A detailed account of the presenting illness, past medical history, and family history was obtained. All children were examined and a
Glasgow meningococcal septicaemia prognostic score was estimated. ${ }^{4}$

Of the 69 cases, 53 were admitted because of the suspicion of meningococcal disease; 16 were not initially thought to have meningococcal disease and were included in the study later when meningococci were cultured from blood or cerebrospinal fluid.

Blood samples were collected at the time of admission. Full blood count including differential white cell and platelet counts were estimated by standard laboratory methods. Serum $\mathrm{C}$ reactive protein concentrations were estimated by laser nephelometry (Beckman Scientific Instruments Ltd). Samples of plasma were stored at $-70^{\circ} \mathrm{C}$ before estimation of total haemolytic complement activity by a quantitative assay (Kallestad Laboratories, Quantiplate).

Bacteriological confirmation of the diagnosis was sought by standard Gram stain and culture of cerebrospinal fluid or blood, or both, either by the detection of meningococcal antigen in blood by latex agglutination and counter immunoelectrophoresis or in cerebrospinal fluid by latex agglutination (Wellcome Diagnostics).

Of the 69 cases, 54 were confirmed by one of the methods outlined above. A further six patients (all of whom had purpuric rashes) had abnormalities in the cerebrospinal fluid consistent with meningitis but had already been treated with antibiotics. These six were considered to have meningococcal disease giving a total of 60 confirmed cases. Each child who had a maculopapular rash alone was confirmed as having meningococcal disease on culture.

Nine other children, all of whom had typical purpuric rashes, were included on clinical grounds. In five children screening tests for meningococcal disease including examination of cerebrospinal fluid showed no evidence of meningococcal disease, but they had all been treated with antibiotics. Three had clinical evidence of meningitis but were considered too ill to have lumbar punctures on admission. One had no evidence of infection and no serological evidence of viral disease, but had a raised $\mathrm{C}$ reactive protein concentration.

Data were analysed with the Biostatistic Analysis Package (C Hetherington 1987) on a BBC B microcomputer. The Mann-Whitney U test was used to assess the significance of differences between groups.

\section{Results}

A total of 69 children were admitted with clinically suspected meningococcal disease, and in 60 it was confirmed by direct or indirect bacter- 
iological methods. Meningococci were cultured from all the children without rashes or whose only skin sign was a maculopapular rash.

Four children had meningitis but no rash. Twenty five had septicaemia without meningitis, and 24 of these had rashes. The remaining 40 children had both rashes and clinical evidence of meningitis (septicaemia and meningitis).

The children were classified according to the type of rash associated with their illness: maculopapular alone, petechial or purpuric, or both, mixed rash (both types), or no rash. The clinical and laboratory data are summarised in the table. There were 39 boys and 30 girls, a sex ratio of 1.3:1. Their ages ranged from 1 month to 14.5 years (median 18 months). There was no significant difference in age between children presenting with maculopapular rashes and those with other types of rash, but those children who did not develop a rash at any stage of their illness were significantly younger than those with classic purpuric rashes $(p<0.05)$ or mixed rashes $(\mathrm{p}<0.01)$.

Nine children had maculopapular rashes alone. Seventeen had mixed rashes with both maculopapular and petechial or purpuric elements, and 38 had typical haemorrhagic rashes. Only five children did not develop a rash at any stage of their illness: four had meningitis and one had septicaemia.

Of the nine children who had maculopapular rashes alone, four had transient rashes that lasted for less than 24 hours after admission and in five the rashes persisted for up to three days after treatment had been started. Eight of these nine children had meningitis. The maculopapular rash was distributed on the trunk alone in six of the nine cases, on the trunk and extremities in two, and on the extremities alone in one (figure).

Sixty of the 69 children were feverish on admission including seven of the nine children who had maculopapular rashes alone; one of the remaining two became feverish after admission. The ninth patient was a teenager who although never feverish had headaches, vomiting, drowsiness, and double vision.

Children with maculopapular rashes had a significantly lower median Glasgow meningococcal septicaemia prognostic score $(p<0.05)$ and significantly higher median plasma haemolytic complement activity $(p<0.05)$ than those with purpuric rashes, but there was no difference between them and patients with any other type of rash. Children who had purpuric rashes on presentation had a significantly lower

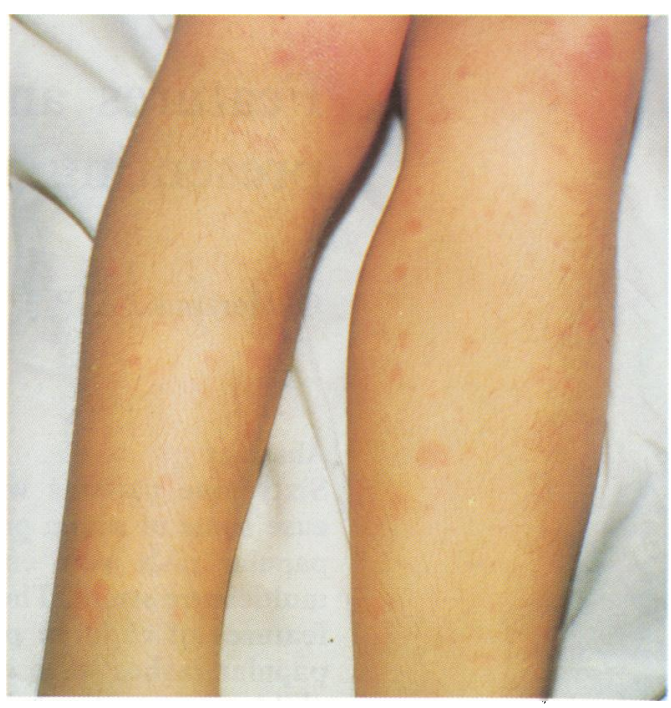

Maculopapular rash associated with meningococcal disease.

median platelet count than patients in any of the other groups (table).

There were no differences among the groups in $\mathrm{C}$ reactive protein concentration, total white cell or absolute neutrophil count on admission, or mortality.

Twelve children remained untreated for periods ranging from eight to 48 hours because the diagnosis of meningococcal disease was not considered. There were three in each of the four groups. The only death among these initially untreated patients had a mixed rash: the child had presented with a maculopapular rash that evolved eight hours later into an extensive purpuric rash.

\section{Discussion}

Previous reports of maculopapular rashes occurring in meningococcal disease have been of isolated cases $^{2}$ or in retrospective reviews. ${ }^{35}$ In this prospective survey of meningococcal disease 64 children (93\%) developed rashes during their illnesses; 26 children (38\%) had maculopapular rashes with or without petechiae or purpura, and nine (13\%) presented with maculopapular rashes alone. If we consider only those children in whom the disease was confirmed bacteriologically, the percentage of children with maculopapular rashes alone rises to $15 \%$.

It has been suggested that meningococcal disease presenting with a maculopapular or petechial rash has a good prognosis and that the

Comparison of type of rash in meningococcal disease with clinical and laboratory variables

\begin{tabular}{|c|c|c|c|c|}
\hline & \multicolumn{3}{|l|}{ Type of rash } & \multirow[b]{2}{*}{$\begin{array}{l}\text { No rash } \\
(n=5)\end{array}$} \\
\hline & $\begin{array}{l}\text { Maculopapular } \\
(n=9)\end{array}$ & $\begin{array}{l}\text { Purpuric } \\
(n=38)\end{array}$ & $\begin{array}{l}\text { Mixed } \\
(n=17)\end{array}$ & \\
\hline $\begin{array}{l}\text { Median age (months) } \\
\text { Median Glasgow meningococcal septicaemia prognostic score } \\
\text { Median platelet count }\left(\times 10^{9} / \mathrm{l}\right) \\
\text { Median haemolytic complement activity in plasma (CH50) (U/ml) } \\
\text { Median white cell count }\left(\times 10^{9} / 1\right) \\
\text { Median neutrophil count }\left(\times 10^{9} / 1\right) \\
\text { Median C reactive protein concentration (mg/l) } \\
\text { No of deaths }\end{array}$ & $\begin{array}{l}9 \\
2 \cdot 5^{*} \\
294^{*} \\
80 \cdot 5^{*} \\
16 \cdot 3 \\
10 \cdot 8 \\
80 \\
0\end{array}$ & $\begin{array}{c}26 \cdot 5 \\
5 \cdot 5^{*} \\
243^{*} \\
65^{*} \\
14 \cdot 8 \\
8 \cdot 9 \\
83 \\
5\end{array}$ & $\begin{array}{r}13 \\
3 \\
299 \\
65 \\
19 \cdot 9 \\
10 \cdot 0 \\
90 \\
1\end{array}$ & $\begin{array}{l}2 \\
\text { Incomplete data } \\
338 \\
130 \\
9 \cdot 1 \\
3 \cdot 5 \\
\text { Incomplete data } \\
0\end{array}$ \\
\hline
\end{tabular}

${ }^{*} \mathrm{p}<0.05$. 
nature of the rash on admission could be used as a prognostic indicator. ${ }^{5}$

Maculopapular rash alone in meningococcal disease has, however, also been reported in fatal cases, ${ }^{6}$ and it is possible that the delay in diagnosis of meningococcal disease with a maculopapular rash alone might contribute to mortality as these children are thought to have viral illnesses and are not started on antibiotic treatment.

In our series none of the children with a maculopapular rash alone died. There was only one child who presented with a maculopapular rash that later progressed to a typical purpuric rash. This girl was observed untreated for eight hours; when her rash was seen to contain some petechiae antibiotic treatment was started. Her rash progressed, she continued to deteriorate, and despite intensive support she died 36 hours after admission.

She had had no evidence of neck stiffness on admission and a low white cell count with relative neutropenia, both of which are poor prognostic factors in typical meningococcal disease. ${ }^{7-10}$ It seems that the same poor prognostic factors apply when children with meningococcal disease present with maculopapular as with any other type of rash. The changing nature and evolution of the rash in meningococcal disease in this child emphasises the need to review children after admission and to report any changes in the nature of the rash.

Platelet count and plasma total haemolytic complement activity were the only laboratory variables that differed significantly between children presenting with maculopapular and purpuric rashes. Thrombocytopenia with or without disseminated intravascular coagulation is a poor prognostic factor, ${ }^{10} 11$ and low haemolytic complement activity in plasma has been associated with rapidly progressive meningococcal disease. 12

It must be noted, however, that despite the significance of the difference in platelet counts and haemolytic complement activity these median values are all within the normal reference range. Children with maculopapular rashes had a significantly lower median Glasgow meningococcal septicaemia prognostic score than those presenting with purpuric rashes. This difference is not entirely explicable on the basis of the different types of rash, because an extending, ecchymotic, or extensive purpuric rash contributes only one point out of a total of 15 on the prognostic score.

Differentiating the maculopapular rash or meningococcal disease from that of a viral infection remains a problem. Diagnosis before haemorrhagic elements develop would permit earlier treatment.

Laboratory variables such as $\mathrm{C}$ reactive protein concentration may be helpful in supporting the diagnosis, but ultimately bearing the diagnosis in mind when attending a feverish child with a rash is of great importance.

One striking point in the history of the maculopapular rash in meningococcal disease is its rapid evolution. If this is important it could be the only clue on admission that would enable a clinical differentiation to be made between the maculopapular rash of meningococcal disease and that of non-specific viral disease. A prospective study to answer this question is in progress.

Careful examination of feverish children with rashes is important. Of the 17 children in our series who had mixed rashes, $12(71 \%)$ had only a few haemorrhagic spots (less than 12) within a predominantly maculopapular rash.

In this study the only child whose rash evolved from being only maculopapular to include haemorrhagic elements died. Her rash evolved eight hours after admission. This is consistent with other such reported cases in which the rash usually evolved within 24 hours of admission. ${ }^{3} 6$ During this critical first 24 hours close observation of the rash and regular application of the Glasgow meningococcal septicaemia prognostic score to detect changes in clinical condition may help to prevent such deaths.

In summary, this is the first prospective study to our knowledge to establish the incidence of meningococcal disease presenting with a maculopapular rash alone. This occurred in nine children ( $13 \%$ of all cases and $15 \%$ of confirmed cases), while 17 (25\%) had mixed maculopapular and haemorrhagic rashes.

Despite its benign appearance the maculopapular rash can still herald a potentially fatal disease. Meningococcal disease must be considered in the differential diagnosis of any feverish child with a maculopapular rash.

1 Brandtzaeg P, Dahle JS, Hoiby EA. The occurrence and features of haemorrhagic skin lesions in 115 cases of systemic meningococcal disease. NIPH Ann 1983;6: $183-90$.

2 Rubenstein R, Esterly N. Meningococcal meningitis with a benign skin rash. Pediatric Dermatol 1986;3:414-6.

3 Carter PE. Meningococcal meningitis. BMF 1990;300:1584.

4 Sinclair JF, Skeoch CH, Hallworth D. Prognosis of meningococcal septicaemia. Lancet 1987;ii:38.

5 Toews WH, Bass JW. Skin manifestations of meningococcal infection. Am $\mathcal{F}$ Dis Child 1974;127:173-6.

6 Baxter P, Priestley B. Meningococcal rash. Lancet 1988; 1166-7.

7 Stiehm ER, Damrosch DS. Factors in the prognosis of meningococcal infection. $\mathcal{f}$ Pediatr 1966;68:457-67.

8 Ansari BM, Davies DB, Boyce JMH. A comparative study of adverse factors in meningococcaemia and meningococcal meningitis. Postgrad Med $\mathcal{f}$ 1979;55:780-3.

9 Leclerc F, Beuscart R, Guillois B, et al. Prognostic factors of severe infectious purpura in children. Intensive Care Medicine 1985;11:140-3.

10 Niklasson P-M, Lundbergh P, Strandell T. Prognostic factors in meningococcal disease. Scan $\mathcal{F}$ Infect Dis $1971 \cdot 3$. 17-25.

11 Khan A, Blum D. Factors for poor prognosis in fulminating meningococcaemia. Clin Pediatr (Phila) 1978;17:680-3.

12 Beatty DW, Ryder CR, Hesse H de V. Complement abnormalities during an epidemic of group B menin gococcal infection in children. Clin Exp Immunol 1986;64: 465-70. 